\title{
Decision support system for integrated management of mycotoxins in feed and food supply chains
}

\author{
H.J. van der Fels-Klerx ${ }^{1,2^{*}}$, C. Liu ${ }^{1}$, M. Focker ${ }^{2}$, I. Montero-Castro ${ }^{3}$, V. Rossi ${ }^{4}$, V. Manstretta ${ }^{5}$, N. Magan ${ }^{6}$ and R. Krska $^{7,8}$ \\ ${ }^{1}$ Wageningen Food Safety Research, Akkermaalsbos 2, 6708 WB Wageningen, the Netherlands; ${ }^{2}$ Wageningen University, \\ Business Economics Group, Hollandseweg 1, 6706 KN, Wageningen, the Netherlands; ${ }^{3}$ IRIS Technology Solutions S.L., \\ Avda. Carl Friedrich Gauss 11, 08860 Castelldefels, Barcelona, Spain; ${ }^{4}$ Università Cattolica del Sacro Cuore, via Emilia \\ Parmense 84, 29122 Piacenza, Italy; ${ }^{5}$ Horta s.r.l., via Egidio Gorra 55, 29122 Piacenza, Italy; ${ }^{6}$ Applied Mycology Group, \\ Environment and AgriFood Theme, Cranfield University, Cranfield, Beds. MK43 OAL, United Kingdom; ${ }^{7}$ Institute of \\ Bioanalytics and Agro-Metabolomics, Department IFA-Tulln, University of Natural Resources and Life Sciences, Vienna \\ (BOKU), Konrad-Lorenz-Str. 20, 3430 Tulln, Austria; ${ }^{8}$ Institute for Global Food Security, School of Biological Sciences, \\ Queens University Belfast, University Road, Belfast, BT7 1NN, Northern Ireland, United Kingdom; ine.vanderfels@wur.nl
}

Received: 2 June 2020 / Accepted: 7 April 2021

(c) 2021 Wageningen Academic Publishers

\section{OPEN ACCESS @ (c) RESEARCH ARTICLE}

\begin{abstract}
Mycotoxins present a global food safety threat of our feed and food. Mycotoxins are toxic metabolites of certain fungi in agricultural products that are harmful to animal and human health. The presence of mycotoxins in these products depends on a variety of management and environmental factors in the field, during storage and/or processing of feed and food commodities. To date, information on mycotoxin management is available, but is not easy to access by supply chain actors. This study aimed to design, build and test a Decision Support System (DSS) that can help decision making on mycotoxin management by various actors along the feed and food supply chains. As part of this, available knowledge and data on mycotoxin prevention and control were collected and synthesised into easy to understand guidelines and tools for various groups of end-users. The DSS consists of four different modules: (a) static information module and (b) scenario analysis module, (c) dynamic module for forecasting mycotoxins, and (d) dynamic module for real-time monitoring of moulds/mycotoxins in grain silos. Intended end-users are all enduser groups for modules (a) and (b); growers and collectors for module (c) and; post-harvest storage managers for module (d). The DSS is user-friendly and accessible through PCs, tablets and smartphones (see https://mytoolboxplatform.com/). In various phases of the DSS development, the tool has been demonstrated to groups of end-users, and their suggestions have been taken into account, whenever possible. Also, a near final version has been tested with individual farmers on the easiness to use the system. In this way we aimed to maximise the DSS uptake by actors along the chain. Ultimately, this DSS will improve decision making on mycotoxin management; it will assist in reducing mycotoxin contamination in the key crops of Europe, thereby reducing economic losses and improving animal and human health.
\end{abstract}

Keywords: food safety, agronomic practices, ICT, end-user, mycotoxin management

\section{Introduction}

Mycotoxins are toxic secondary metabolites produced by certain filamentous fungi in agricultural products, such as wheat, barley and maize, peanuts and figs, which affect animal and human health. The presence of the fungi in the crops and the production of mycotoxins depends on a variety of management and environmental factors in the field, during storage and/or processing. Contamination of feed and food commodities with mycotoxins is a worldwide food safety challenge (Atanda et al., 2012; Krska et al., 2016; López-García, 2010; Lopez-Garcia et al., 1999). All actors in the entire production chain should apply and show utmost adherence to best management practices in order 
to limit mycotoxin contamination of the final feed and food products to the lowest level achievable. This requires integration of up-to-date pre- and post-harvest knowledge to develop a framework for providing practical solutions to farmers, storage managers and processors.

There is a wealth of available knowledge of intervention strategies reported over the last 30 years to minimise mycotoxin contamination, especially of staple food and feed chains, collected from laboratory experiments (Alonso et al., 2017; De Rijk et al., 2015) or field trials (Del Ponte et al., 2007; Garcia-Cela et al., 2018, 2019, 2020; Landschoot et al., 2012; Van Asselt et al., 2012a; Van der Fels-Klerx et al., 2012a). Also, efforts have been made to develop forecasting models for mycotoxins in the various types of grains. As examples, such models include deoxynivalenol in pre-harvest wheat (Del Ponte et al., 2005; Franz et al., 2009; Klem et al., 2007; Liu et al., 2018; Schaafsma and Hooker, 2007; Van Der Fels-Klerx et al., 2010), various mycotoxins in pre-harvest maize (Battilani and Leggieri, 2015; Battilani et al., 2013; Chauhan et al., 2015; Van Asselt et al., 2012b), as well as for stored grains post-harvest (Aldars-García et al., 2016; Garcia-Cela et al., 2019, 2020).

However, this information on mycotoxin management strategies has seldom been made available, by integrating the components with ICT tools, for application by stakeholders in practice in the pre-harvest, post-harvest and/or processing phases of key staple cereal supply chains. Four years ago, international project groups were formed to tackle this gap with two large European funded projects: MycoKey (www.mycokey.eu) and MyToolBox (www. mytoolbox.eu). In the course of the MycoKey project, an app was developed and integrated in the Akkerweb platform (www.akkerweb.eu) generated by Wageningen University \& Research to provide mycotoxin risks for cereals and maize globally. The mission of the MyToolBox project was to mobilise and integrate existing knowledge with novel findings to provide a vehicle to practically translate and implement this knowledge into ICT tools for use along feed and food chains (Krska et al., 2016).

The aim of this study was to make existing knowledge on mycotoxin prevention and control strategies available to end-users along the chain via a user-friendly decision support system (DSS). The study was composed of two parts with the following sub-objectives: (a) to collect available knowledge on mycotoxin prevention and control along the chain and to synthesise this into easy-to-understand guidelines and tools for the end-user, and (b) to design, build and test an integrated, dynamic electronic (E-) platform for mycotoxin management to support decision making for actors along the chain. To date, no such fully integrated DSS is available for mycotoxin minimisation and control along the entire supply chain.

\section{Materials and methods}

The study focused on the most relevant mycotoxins in the cereal (wheat, barley, maize), dried fig and peanut supply chains in Europe. These crops are major feed and food commodities around the world which are vulnerable for mycotoxin contamination. Mycotoxins considered were selected based on their occurrence, toxicology and legislative limits in Europe, and included: deoxynivalenol in (durum) wheat and barley, aflatoxins and fumonisins in maize, and aflatoxins in dried figs and peanuts. The DSS consists of different modules for various groups of end-users, including:

(a) a static information module for all end-user groups;

(b) a scenario analysis module for pre-harvest agronomics for all end-users;

(c) a dynamic module for forecasting mycotoxins in preharvest cereals for growers and buyers; and

(d) a dynamic module for real-time monitoring of moulds/ mycotoxins in silos (silo management module) for postharvest storage managers.

For using the two dynamic component modules (c and d), information from the end-user is needed and, therefore, these require pre-registration (see Section on DSS development). The four different DSS modules are described in more detail in the following sections.

\section{Static information module}

Available guidelines for Good Agricultural Practices (GAP) and Good Manufacturing (GMP) practices as well as protocols for Hazard Analysis and Critical Control Points (HACCP) for prevention and control of mycotoxins in Europe were gathered for the mycotoxins and supply chains considered in this study. All available data and information from the guidelines and protocols as well as from scientific literature and regulatory documents, were synthesised and evaluated. Next, the information was synthesised and summarised into easy-to-understand information sheets and flow charts. The information sheets contain information on: (a) background of the problem, (b) advice, and (c) further reading for the end-user. Guidelines were developed separately per crop, and divided into pre-harvest, post-harvest, processing, regulation \& control, and safe use options.

Pre-harvest guidelines were specified separately for each of the different management practices, such as tillage and crop rotation, for each of the five crops (wheat, barley, maize, figs, peanuts) under consideration (APC, 2011; Bruns, 2003; CAC, 2003, 2004, 2008; EC, 2006a; Food Standards Agency, 2007; Savić et al., 2020). For example, to reduce deoxynivalenol contamination in wheat, grain farmers are provided with background information and advice regarding the choice of the cultivar, tillage method, crop 
rotation, fungicide and/or biopesticide use and harvest practices, as well as the most relevant references for further reading.

Post-harvest guidelines were divided into two parts: storage, and sampling \& analysis. Storage guidelines present information and advice on storage preparation, the reception of the crop, the drying of the crop, and subsequent storage, for each of the five crops separately. Also, parameters that should be monitored during storage, such as the temperature, moisture content, and the presence of fungi, insects and rodents, are described. Guidelines about sampling and analysis based on the European Regulations as well as based on research projects were summarised (EC, 2006a,b,c, 2009; Focker et al., 2019; Stadler et al., 2018).

Processing guidelines were developed for the three grain crops (wheat, barley, maize) and consist of flowcharts of different processing methods with, for each step of the process, information about how the processing step affects the concentrations of several mycotoxins. For example, flowcharts of dry-milling and wet-milling of wheat were developed, as well as the processes of the production of, for example, rusks, biscuits, bread, pasta or beer.

Regulation and control consists of a search tool in which the crop can be chosen, together with the intended use of the crop (feed or food) and, then, the relevant maximum limits for mycotoxin presence in the particular crop are provided to the end-user.

The safe use option part of the static information module provides options for both contaminated wheat and maize.

\section{Scenario analysis module}

The scenario analysis function is an interactive tool in the pre-harvest part of the DSS for optimising strategic choices concerning pre-harvest crop management so as to limit deoxynivalenol contamination of wheat. It provides opportunities for the cereal growers and other stakeholders to evaluate what would be the deoxynivalenol contamination probability (low, medium high) in wheat for several combinations of agricultural practices and weather conditions. In this tool, the end-user is asked to choose a combination of agricultural practices, e.g. the previous crop, the soil cultivation method, the relative resistance of the wheat variety against Fusarium spp., the fungicide application, and the weather conditions. With these choices, the probability of deoxynivalenol contamination is calculated based on historical data, and presented to the end-user in deoxynivalenol risk classes, as low $(<500 \mu \mathrm{g} / \mathrm{kg})$, medium $(\geq 500 \mu \mathrm{g} / \mathrm{kg}$ and $<1,250 \mu \mathrm{g} / \mathrm{kg})$ or high $(\geq 1,250 \mu \mathrm{g} / \mathrm{kg})$. The end-users can either estimate the deoxynivalenol contamination by changing only one agricultural practice or keep all practices the same and test the effect (what-if scenario) of different weather conditions. This scenario analysis tool can be used for long-term (next season) mycotoxin management. It has been developed for deoxynivalenol in wheat only, since there was sufficient data availability for this mycotoxin-cereal system. If, in the future, more data become available for the other mycotoxincereal combinations, the scenario analysis tool can be extended for these toxins and/or cereals.

\section{Dynamic module for forecasting mycotoxins}

The DSS dynamic module for forecasting mycotoxins in the pre-harvest stage focuses on deoxynivalenol contamination in (durum) wheat and barley, and aflatoxins and fumonisins contamination in maize at the field level in Europe. For these mycotoxins-cereal combinations, various forecasting models that predict toxic levels at harvest have been developed and, in most (but not all) cases, published. These models use input data on weather during the cereal growing season, cereal phenology dates related to seed sowing, flowering and harvest, and - in some cases - agronomic information, such as cereal variety, tillage method and the previous crop cultivated in the particular field. In previous studies, forecasting models have been developed for deoxynivalenol in wheat and barley for different European regions, such as for Germany (Birr et al., 2019), Belgium (Landschoot et al., 2013a,b), Italy (Rossi et al., 2007), the Netherlands (Liu et al., 2018; Van der Fels-Klerx et al., 2010), and North-West Europe (Van der Fels-Klerx et al., 2012b). Some of the models are empirical, i.e. defined by mathematical relationships between input and output data, whereas others are mechanistic, i.e. consider the biological processes leading to the development of toxin producing fungi and toxin accumulation (Rossi et al., 2003a, 2007). In the current study, efforts were made to both retrieve the published models and to develop new models or elaborate upon the existing ones. For published models, model code was deduced from the scientific articles. When such inferences were not possible, authors of the published models were directly contacted and asked to make the model codes available for inclusion in the DSS. Next, all model algorithms were coded into the appropriate language (e.g. R or java) for integration into the E-platform, and a model ensemble was developed to integrate results from the (eleven) different models to predict deoxynivalenol in wheat and barley at harvest. Ensembling techniques were used to combine the predictions of a group of models (Birr et al., 2019; Landschoot et al., 2013a,b; Liu et al., 2018; Rossi et al., 2003a,b, 2007; Van Der Fels-Klerx et al., 2010, 2012c) to generate more accurate predictions. All these wheat and barley models were validated with external sets of data that were not used in the model development. The detailed validation methods and performance of the individual models are referred to the original publications. Ensemble techniques reduce model selection bias that can result in 
model selection uncertainties (Collins et al., 2011; Tebaldi and Knutti, 2007). The aim of the ensemble modelling in the current study was to use all available models for prediction of deoxynivalenol in wheat and barley in Europe with their predictions, weighted to the relevance of the particular cereal field for which the forecast is requested by the end-user.

As part of the MyToolBox project, prediction models for fumonisins and aflatoxins in maize in Europe have been developed. For maize, two types of published models to predict the aflatoxins (Battilani and Leggieri, 2015; Chauhan et al., 2015) and fumonisins (Maiorano et al., 2009; Van Asselt et al., 2012b) contamination class were used as the starting point. These mechanistic models first predict the fungal infection risk index based on biological processes including fungal infection stages (e.g. dispersal and germination), maize growth stages, micro-climatic parameters and insect damage, and then statistically relate toxin level to the fungal risk index (Battilani et al., 2013; Chauhan et al., 2015). Using these approaches as the basis, a Bayesian Network model was developed by using the fungal risk index in each maize growing period to predict the mycotoxin contamination in two classes, either low ( $\leq 10 \mu \mathrm{g} / \mathrm{kg}$ for aflatoxins and $\leq 1000 \mu \mathrm{g} / \mathrm{kg}$ for fumonisins) and high $(>10 \mu \mathrm{g} / \mathrm{kg}$ for aflatoxins and $>1000 \mu \mathrm{g} / \mathrm{kg}$ for fumonisins) (Liu et al., 2021). The particular country was divided in grids of $25 \mathrm{~km}$ by $25 \mathrm{~km}$ squares, according to grids defined by the Joint Research Centre (JRC) Agri4cast data portal (https://agri4cast.jrc.ec.europa.eu/DataPortal/). Weather data were retrieved from the Agri4cast JRC portal and used to feed the models. All calculations were carried out by using $\mathrm{R}$ (version 3.5.0).

A routine was developed for retrieving weather data throughout Europe and calculating the input values for the parameters of each of the mycotoxin forecasting models. Input on the agronomics of each field was retrieved by (manual) input from the end-user, after registration, via the front-end of the DSS. All input data were collected and stored in a temporary database, for running the forecast models, at the request of the user.

\section{Dynamic module for silo management}

The monitoring of cereals and nuts stored in silos is a key phase in the value chain to limit mycotoxin contamination. Temperature and relative humidity $(\mathrm{RH})$ are often measured periodically in stored commodities in silos. To further enhance post-harvest silo management and allow for timely remedial actions to reduce the initiation of mould growth and mycotoxin contamination due to wet pockets in the silo, real time monitoring of temperature and RH is needed. A real-time dynamic module for post-harvest management of both cereals and nuts was developed and integrated in the DSS post-harvest module. Temperature and RH data were used to develop boundary models of fungal growth and mycotoxin production in the silo to facilitate the determination of the relative risk of specific mycotoxins exceeding the existing EU regulations (Garcia-Cela et al., $2018,2019,2020)$. These models were recoded into one coding language for integration into the DSS.

In a related study, respiration $\left(\mathrm{CO}_{2}\right)$ of grain and associated mycoflora were shown to be an important abiotic parameter, in addition to temperature and $\mathrm{RH} . \mathrm{CO}_{2}$ changes occurred much earlier than temperature and $\mathrm{RH}$ changes and provide a more sensitive indicator than the other two parameters (Mylona et al., 2012). An integrated sensor system was developed and tested to monitor, in real time, $\mathrm{CO}_{2}$, temperature and $\mathrm{RH}$ in silos. These sensors gather real time data through a simple-to-install design that can be integrated into silos of the food and feed processing industries (more information on the sensors can be found on: www.gescaser.com). Data collected by these sensors are linked to the models in the DSS for real-time prediction of mould growth and presence/absence of the specific mycotoxin(s) in the silos. In the design phase of the electronics of the sensor system, the ATEX compliance of the sensors for application in such storage facilities was taken into account, to avoid the risk of sensors catching fire.

Each sensor system includes the following elements:

- Power source. The power source for the system was designed to minimise total power consumption by switching between monitoring with each sensor in sequence thus minimising the heat generated during the measurements. The electronic design was also integrated with a wireless module that allowed the transmission of the measured signals to a remote control module either via wireless or physically cabled.

- Multiplexer board. The multiplexer board was used to manage and switch between the sensor nodes in sequence providing the data on actual $\mathrm{CO}_{2}$, temperature and RH.

- Nodes. Each cable/line contained a string of four sensor nodes with each node containing the integrated $\mathrm{CO}_{2}$, temperature and $\mathrm{RH}$ sensors. The quantity of nodes per cable/line is limited by the total power consumption.

\section{DSS development}

The technical development of the DSS consisted of three parts, including: (1) system architecture and specifications, (2) development, and (3) verification and validation. The system architecture consisted of five main blocks: (a) the API (Application Programming Interface) for weather forecasting and mycotoxin predictions in the field (hosted by HORTA), (b) the API for fungal growth or toxin appearance inside a silo containing the harvested cereals or peanuts, (c) the sensor data acquisition software, (d) the end user application, and (e) the servers that combine and integrate 
data and blocks (Figure 1). For the part of the DSS related to the pre-harvest forecasting of deoxynivalenol in wheat and barley, the end user application (d) gathers information from the users' fields, such as the crop grown, agronomic (management) information, the location of the field, the dates of the growth phases of the cereal, and the fungicide spays applied to the field (Table 1). These data need to be entered by the end-user, after registration, and are stored for future use by the same person.

This information is sent via APIs to the weather forecasting and mycotoxin prediction software hosted by HORTA, which in turn sends the related mycotoxin predictions via APIs to the platform. Predictions are stored in the platforms' server so that the user can access the weather conditions and their possible effects on the mycotoxin contamination.

The dynamic silo management module of the DSS uses data acquired by the sensors in the silo, including temperature, $\mathrm{RH}$ and $\mathrm{CO}_{2}$ values. These data are collected via software, and combined with other data related to the silos, including data related to the structure of the installation (number of silos, number of lines, number of sensors) and the grain type stored. The latter data are entered by the end-user, after registration, and are stored for future use by the same person. End-user and sensor data are used to run the prediction models; the outcomes being predictions of the fungal/toxin status of the grain in the silo, which are shown to the end-user. The output of the models is stored in the database, so it can be further displayed in a grid-like silo layout. In this layout the user can check the current status of the sensors (with a colour code), the probability of growth of the fungi that can be present in the commodity being stored in the silo, and the probability of the appearance of any mycotoxin that the fungi present may be producing and the at-risk area within the silo.

The development of the DSS platform was performed by following the Scrum methodology (Schwaber, 2004; Schwaber and Beedle, 2002).

Table 1. Data required from the end-user to generate the preharvest mycotoxin predictions for deoxynivalenol in wheat and barley, using the dynamic forecasting tool.

$\begin{array}{lll}\text { Agronomic data } & \text { Location } & \text { Dates } \\ \text { Crop type } & \text { Country } & \text { Sowing date } \\ \text { Previous crop } & \text { Latitude } & \text { Heading date } \\ \text { Crop variety } & \text { Longitude } & \text { Flowering date } \\ \text { Soil tillage } & \text { Nearest weather } & \text { Harvest date } \\ \text { Growing area } & \text { station } & \text { Fusarium Head Blight } \\ & & \text { (FHB) spray dates }\end{array}$

The development included the following activities: setting up of the users' authentication module (registration, authentication, profiling); creation of the data flows and configuration of the databases; development of the inside components (coding of model formulas) and the outside components (dynamic models via web services); development of the front end Graphical User Interface (GUI) for data entry and data viewer for different endusers with restricted access, i.e. registered users and administrators; interoperability testing among dynamic models (data exchange testing); setting up of the static pages; development of info-graphic and visual reporting tool as interpretation guide, ensuring easy to interpret information for users; optimisation work and scale up of the platform.

All data gathered from the end-users are owned and archived by the platform developers under strict General Data Protection Regulation (GDPR) (EC, 2016) and European Union Agency for Cybersecurity (ENISA) recommendations on techniques, including cryptographic hashes, data encryption, tokenization, masking and so on (ENISA, 2019). Potential safety threats, either internal and external, are minimised through a privacy-by-design approach where no data will be used for research or for other purposes. In addition, data minimisation principles in the design and development phases, data confidentiality guarantee through standard cryptographic principles, and direct control provision to users over their data were included.

The iterative verification and validation of the different versions of the E-platform was performed by an internal team of testers (from the three organisations involved). Data flows and API for the communication between the five main blocks (API for weather forecasting and mycotoxin predictions in the fields prior to harvesting; API for fungal growth or toxin appearance inside grain silos, the sensor data acquisition software, the end user application, and the server that puts all the data together) of the DSS platform were tested and validated. Furthermore, each part of the DSS was tested several times during the four years of development by the MyToolBox project partners and by different groups of external end-users throughout Europe. To this end, the DSS was presented at several stakeholder meetings, where attendees were shown and trained to use the E-platform, and asked for feedback and suggestions for improvements. These stakeholder meetings were organised by a MyToolBox partner who was not involved in the DSS development, to give an external and fresh view in summarising these feedbacks. A specific and detailed test on the use of the DSS was conducted in the wheat season 2018-2019, with two farmers involved in Italy, two in the Netherlands, and one in Austria, focusing on the static information module, the scenario analysis tool and the pre-harvest dynamic module. Tests of the post-harvest 


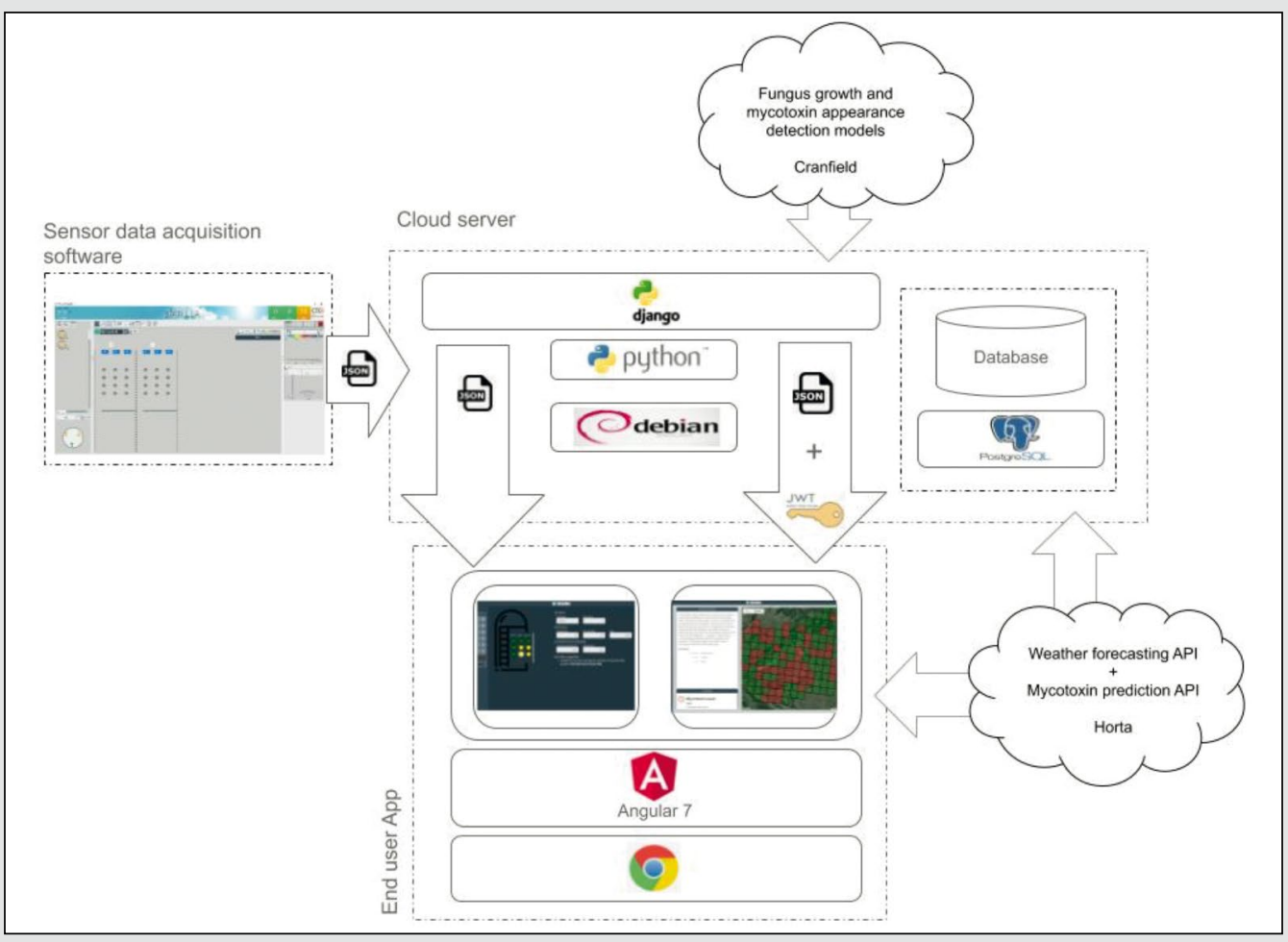

Figure 1. DSS architecture diagram.

system encompassing the sensors and the digital tool were conducted in Barilla facilities in Parma, Italy for wheat and maize, as well as in the Academy of State Administration of Grain in China (ASAG) for peanuts. Pilot silos of 2-2.5 tonnes were monitored for several months including the simulation of water ingress events, to measure in real time the response of the sensors.

\section{Results and discussion}

\section{Static module}

The static information module of the DSS does not require registration to the platform to be accessed: it is openly available at: https://mytoolbox-platform.com/. The content is organised into five sections: (1) pre-harvest, (2) postharvest, (3) processing, (4) regulation and control, and (5) safe use options. Figure 2 presents the front-end of the starting page of the static part of the DSS.

The information in each section is then further organised into sub-sections, changing based on the crop selected by the user. In each section, the information is organised in boxes, which contain information on background, the advice, and links for useful further reading for the enduser (Figure 3).

\section{Pre-harvest}

The pre-harvest section displays information on the techniques to be applied in the field cropping in order to reduce mycotoxin contamination of the commodity. Pre-harvest information was gathered for each of wheat, barley, maize, figs and peanuts, separately. For each crop, information is displayed in boxes, representing the relevant sub-sections. Clicking on each of the boxes results in the information being displayed.

For wheat, pre-harvest static information is organised in nine sub-sections, each displaying the relevant content aimed at the reduction of the presence of deoxynivalenol (and other Fusarium toxins, such as zearalenone): (1) cultivar (see also Figure 3 for an example); (2) tillage and crop debris; (3) crop rotation; (4) fungicide use; (5) biopesticide use; (6) scenario analysis (providing the user a tool for simulating deoxynivalenol contamination in wheat based on the selection of several agronomic and weather related parameters); (7) harvest practices; (8) forecasting (which re-addresses the information used in the pre-harvest dynamic forecasting module); (9) regulations, which displays mycotoxin limits for the selected commodity. For barley, preharvest static information for reduction of deoxynivalenol is organised in a similar manner as for wheat, but in seven sub-sections. Bio-pesticides and the scenario analyses tool were not included because of too limited data for barley. 


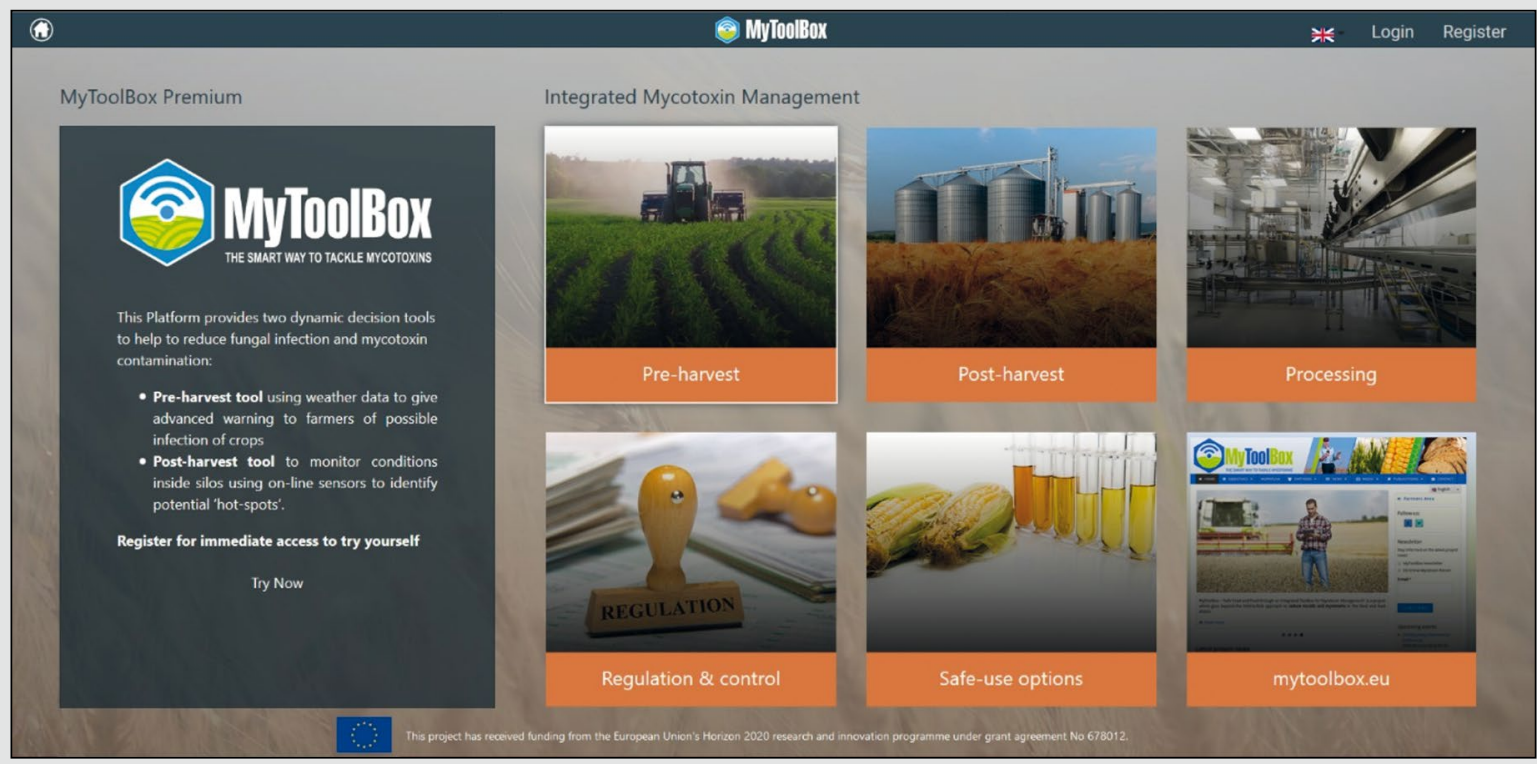

Figure 2. DSS landing page, displaying static content sections and the link to the MyToolBox project website.

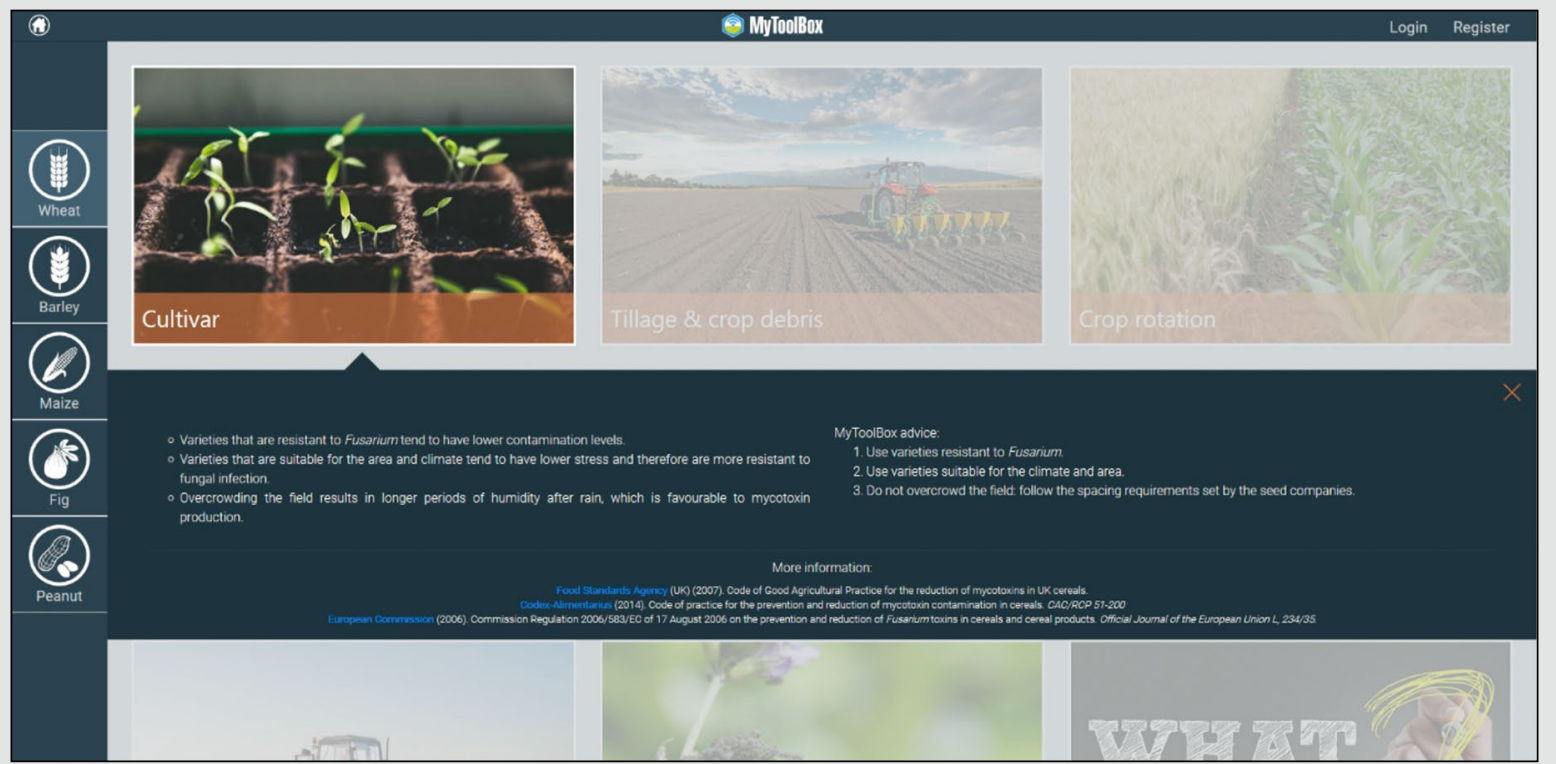

Figure 3. DSS static module: example for information, advice and relevant useful links of the DSS, related to wheat cultivar (note that information related to the other agronomic practices is faded, since Cultivar has been selected).

For maize, pre-harvest static information is organised in eight sub-sections, each displaying the relevant content aimed at the reduction of aflatoxins and fumonisins in this crop: (1) cultivar; (2) sowing date; (3) irrigation; (4) fungicide use; (5) weed/pest control; (6) plant nutrition; (7) harvest practices; (8) biopesticides. For figs, pre-harvest static information is organised into five sub-sections, each displaying the relevant content aimed at the reduction aflatoxins in this commodity: (1) Orchard preparation;
(2) Dust avoidance; (3) Caprifigs; (4) Integrated pest management; (5) Harvest preparation. For peanuts, preharvest static information is organised into eight subsections, each displaying the relevant content aimed at the reduction of aflatoxins: (1) site selection; (2) land preparation; (3) cultivar selection; (4) planting regime; (5) biocontrol; (6) pest and disease management (excl. biocontrol); (7) irrigation; (8) estimating crop maturity. 


\section{Post-harvest}

The post-harvest static section displays information on the techniques to be applied after crop harvest, in order to reduce mycotoxin contamination of the commodity during storage as well as information related to representative sampling and analysis for mycotoxins. For each crop, information is displayed in boxes, representing the relevant sub-section (Figure 4). Clicking on each of the boxes, the information is displayed. Post-harvest information was gathered separately for each of wheat, barley, maize, figs and peanuts.

For wheat, barley and maize, post-harvest static information is organised in two sections: (1) Storage, displaying information on storage preparation, reception of the commodity, drying and storage, (2) representative sampling and analysis.

For figs, post-harvest static information is organised in seven sections: (1) fig collection, (2) drying, (3) transportation, (4) sorting, (5) fumigation, (6) storage, (7) representative sampling and analysis of mycotoxins. For peanuts, postharvest static information is organised in seven sections: (1) harvesting of the crop, (2) sun-drying, (3) hot-air drying, (4) sorting, (5) shelling, (6) storage, (7) representative sampling and analysis of mycotoxins.

\section{Processing}

The Processing section addresses the reduction or concentration of mycotoxin contamination during the commodity processing steps for the production of several feed and food end-products. For the selected processes, this section displays the processing scheme, highlighting the steps in which mycotoxin contamination has been studied, and the effects of the particular processing step on mycotoxin levels (which can be seen by clicking on the step in the flow diagram), and providing links to scientific literature on mycotoxin reduction or increase during the particular processing step (Figure 5). The Processing section was developed for each of wheat, barley and maize, and for several derived feed and food products within each of these grain types. For wheat, displayed processes are: (1) dry-milling, (2) wet-milling, (3) bread, (4) biscuits, (5) rusks, (6) pasta. For barley, displayed processes are: (1) feed, (2) food, (3) brewing (including malting process) of beer. For maize, displayed processes are: (1) dry-milling, (2) wet-milling, (3) feed and bioethanol.

\section{Regulations \& control}

The Regulation \& control section displays the limits for the maximum presence of mycotoxins for each commodity, according to EU legislation (CAC, 2003, 2004, 2008; EC, 2006a,b,c, 2009). When entering this section, a search tool is displayed, which allows the selection of the crop, the purpose of the crop (feed or food production) and the mycotoxin of interest. The tool displays mycotoxin legal limits and provides a link to the reference legislation.

\section{Safe use options}

The safe use option section addresses the possible use of contaminated batches of a commodity. This section displays the possible use and processing schemes for

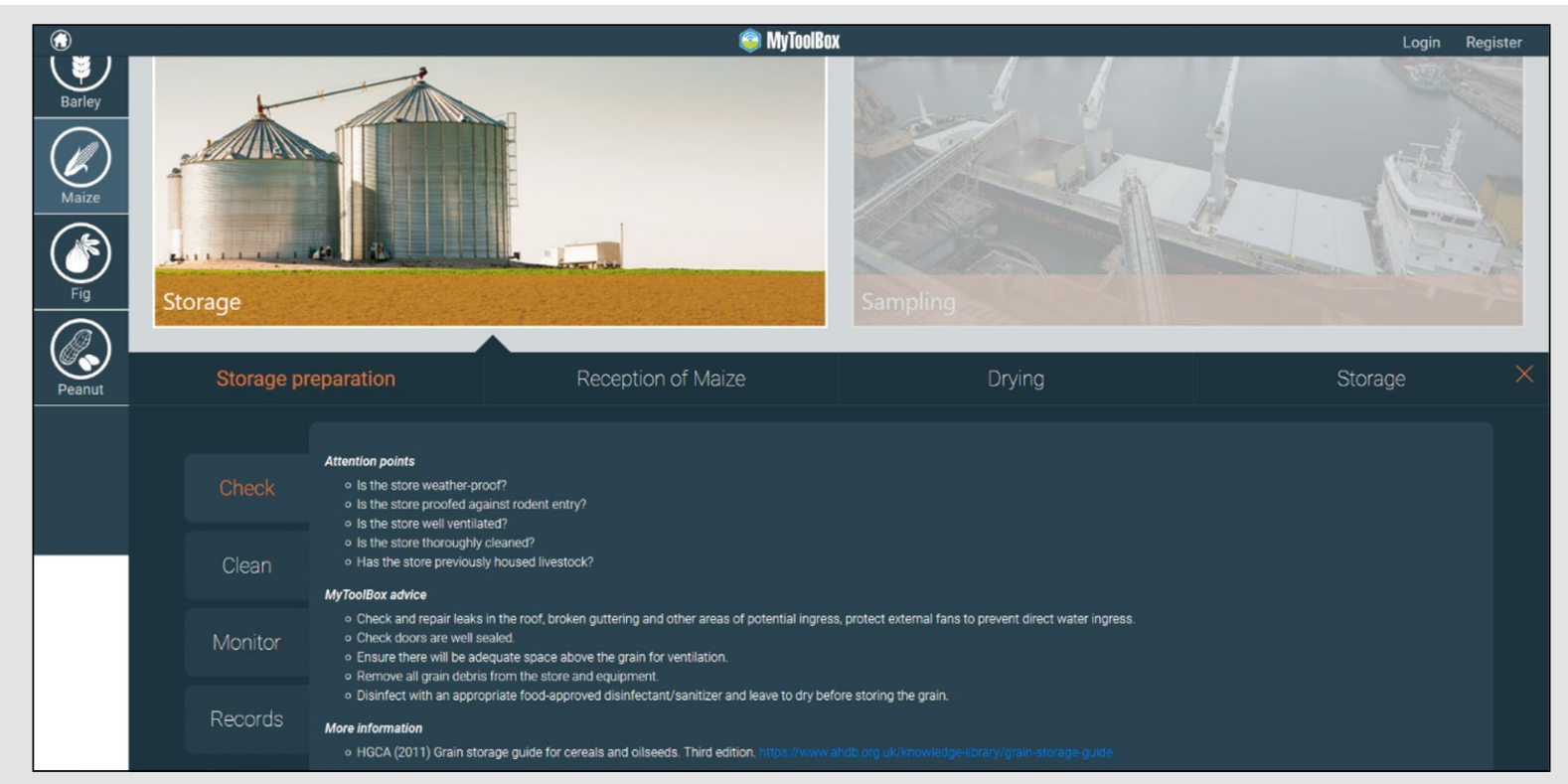

Figure 4. DSS static module: post-harvest section for storage of maize, as example (information on sampling not highlighted since storage has been selected). 


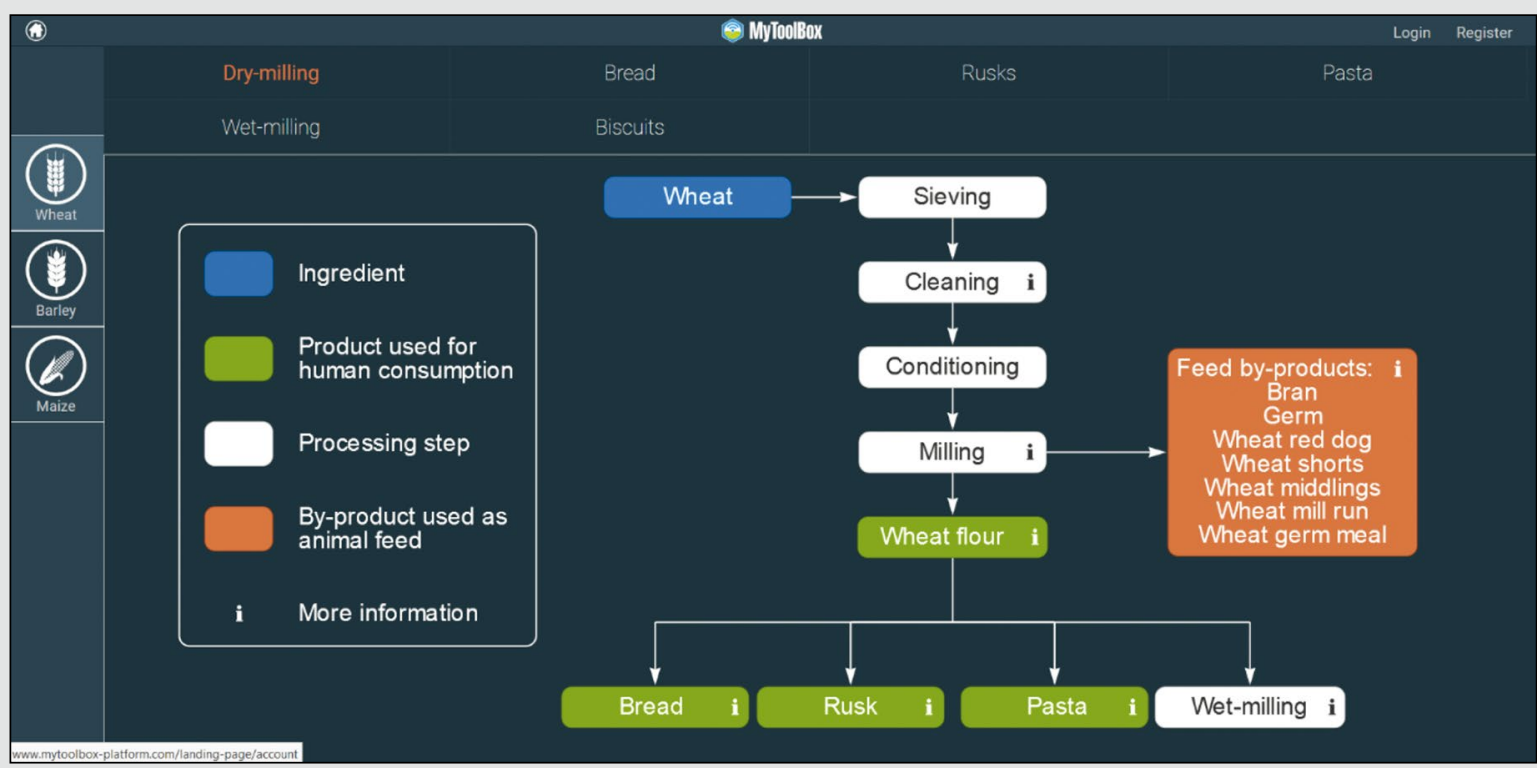

Figure 5. DSS static module: processing section for wheat.

contaminated batches, highlighting the steps in which mycotoxin contamination has been studied, and reporting literature information (Figure 6). The safe use option section was developed for wheat and maize.

\section{Scenario analyses}

With the scenario analysis tool, the end-user can evaluate the impact of different agronomic variables and weather on the deoxynivalenol contamination in wheat, and answer the question 'what would be the deoxynivalenol contamination class if I change to a different management practice in the next growing season?' The end user can select one of the options in the variables previous crop, cultivation, resistant variety, fungicide use and weather. An example is illustrated in Figure 7. The combination of cereals as previous crop to wheat cultivation at the same field (previous crop), ploughing (ploughing), the use of a wheat variety with a medium resistance against Fusarium spp. infection (resistant variety), one fungicide

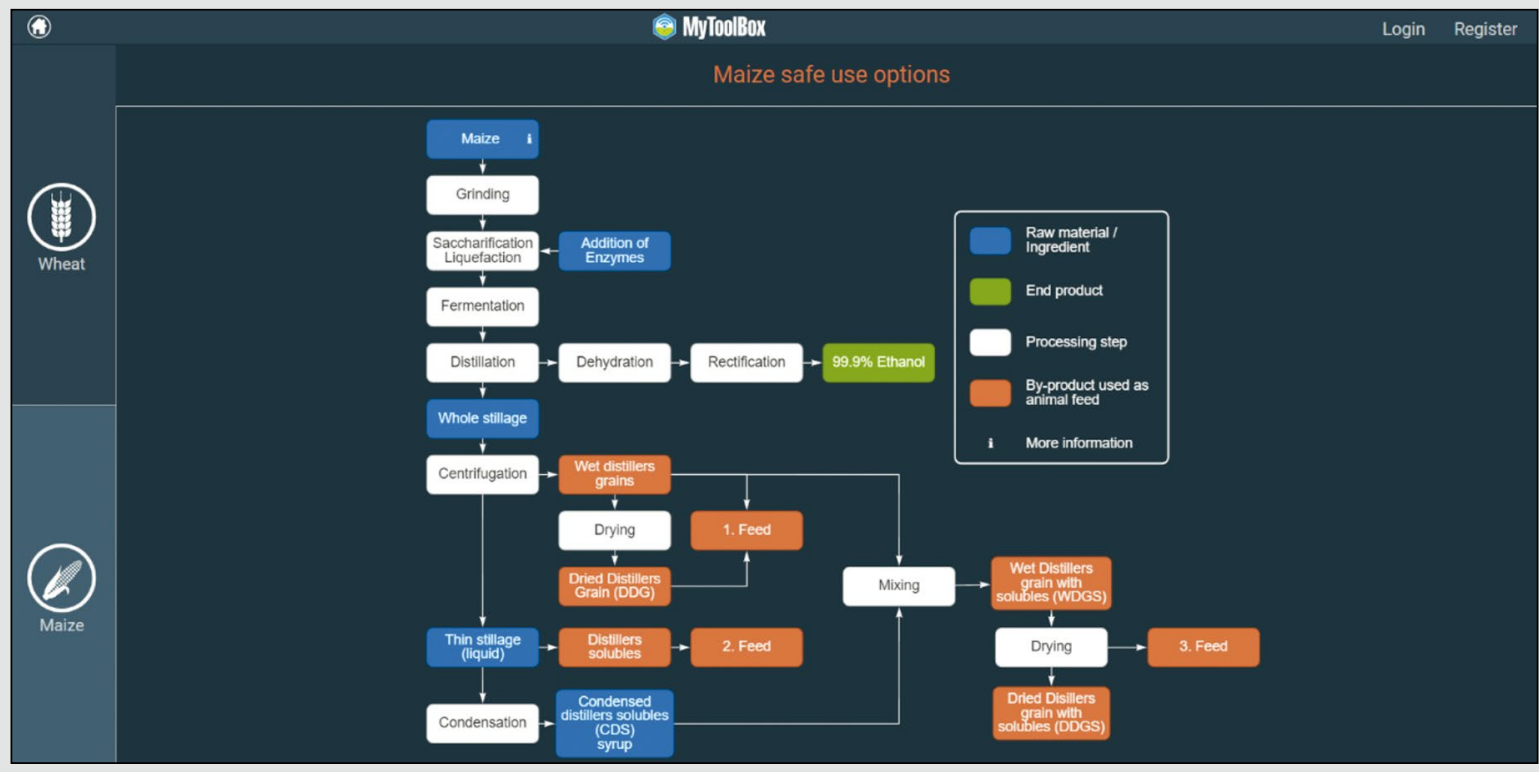

Figure 6. DSS static module: safe use option section for maize. 


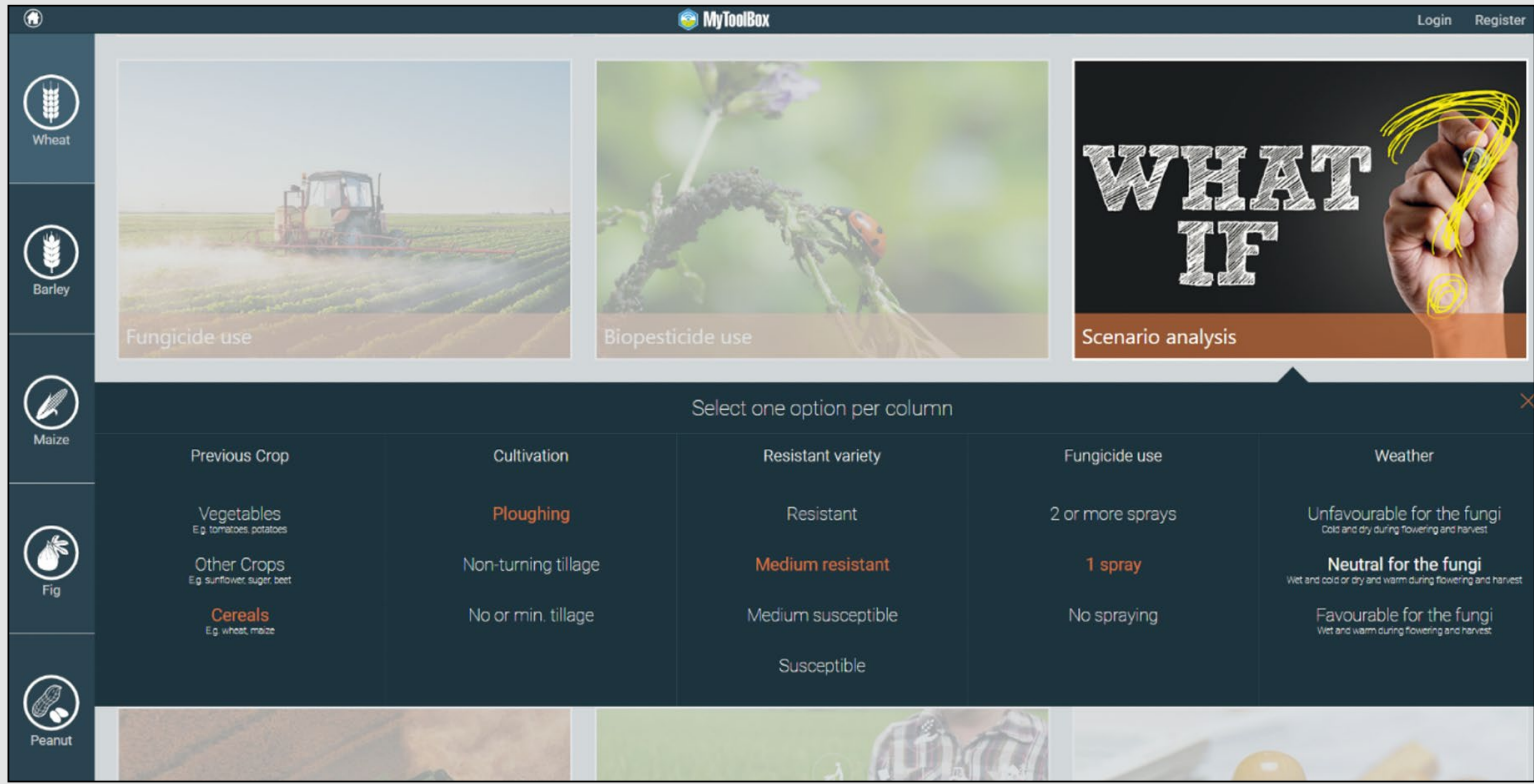

Figure 7. DSS static module: scenario analysis tool with, as example, the agronomic and weather factors selected (Note that other information - not selected by the user - is faded).

spray for Fusarium Head Blight (FHB) at flowering time (fungicide use), and neutral weather conditions during flowering (weather) results into a medium risk according to the scenario analysis tool. This means an expected deoxynivalenol contamination level of between $500 \mu \mathrm{g} /$ $\mathrm{kg}$ (corresponding to the EC legal limit for bread, pastries, biscuits, cereal snacks and breakfast cereals) and 1,250 $\mu \mathrm{g} /$ $\mathrm{kg}$ (corresponding to the EC legal limit for unprocessed normal wheat) at harvest (EC, 2006c). A low risk implies a deoxynivalenol contamination of $<500 \mu \mathrm{g} / \mathrm{kg}$ and a high risk a contamination at $>1,250 \mu \mathrm{g} / \mathrm{kg}$ at harvest.

\section{Forecasting mycotoxins}

The DSS dynamic part is composed of one module for the pre-harvest prediction of mycotoxins, and one for postharvest silo management. To access the dynamic modules of the DSS, the user is requested to register to use the platform. At first access, the user needs to fill in the requested details in the registration module. Each time the user wants to assess his own fields or silos, he/she needs to login.

The pre-harvest forecasting module gives the user the possibility to register his/her own grain fields and to receive predictions for the deoxynivalenol contamination class in each field, on the basis of agronomical inputs and weather data. The structure of the pre-harvest forecasting tool is displayed in Figure 8. Briefly, the system uses weather forecast data (from the APIs) and agronomic parameters, entered by the end-user to feed into the mycotoxin forecasting models. Based on the inputs, the mycotoxin model provides a prediction of the contamination class. The pre-harvest forecasting tool was developed for deoxynivalenol in wheat, durum wheat and barley, and for aflatoxins and fumonisins in maize. The performance of newly developed wheat and barley models for the MyToolBox project were good with the accuracy ranging from 80 to $86 \%$ (Liu et al., 2018).

When logged in, the user has the possibility to see the list of his/her fields, and to create a new field, if needed. When creating a new field, the user needs to enter field-specific data, which vary according to the crop selected (Figure 9). For wheat, durum wheat and barley the user must enter: (1) name of the field, (2) crop, (3) previous crop, (4) crop variety, (5) soil tillage, (6) growing area, (7) country, (9) latitude, (10) longitude, (11) weather station preferred. For input from (2) to (11) the user selects the proper options his/her field from dropdown menus. An explanation of the different input categories is displayed when clicking on an 'information' button page. For maize, the user needs to enter inputs (1), (2), (7), (8) and (9).

For wheat, durum wheat and barley, once the user has set up his/her field, the possibility exists to add relevant crop development dates (sowing, heading, flowering, harvest, and spray dates). Weather forecasts and mycotoxin predictions are displayed, together with a summary of the main field-specific information (Figure 10). For wheat, durum wheat and barley, the probability for deoxynivalenol contamination in the field is given, classified in three classes, being: green $(\leq 500 \mu \mathrm{g} / \mathrm{kg})$, yellow $(500-1,250 \mu \mathrm{g} / \mathrm{kg})$ and 


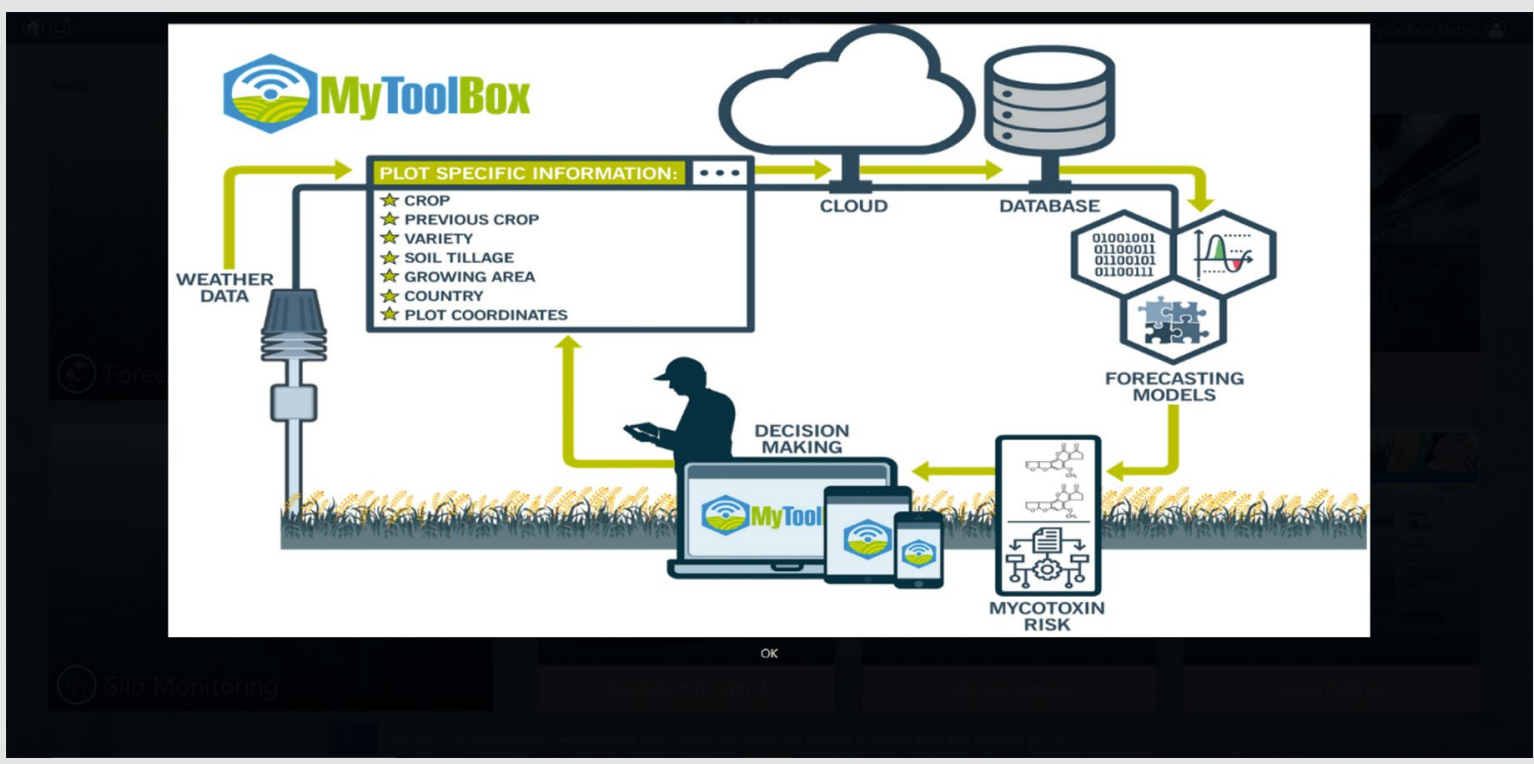

Figure 8. DSS pre-harvest forecasting tool: data and knowledge flow chart.

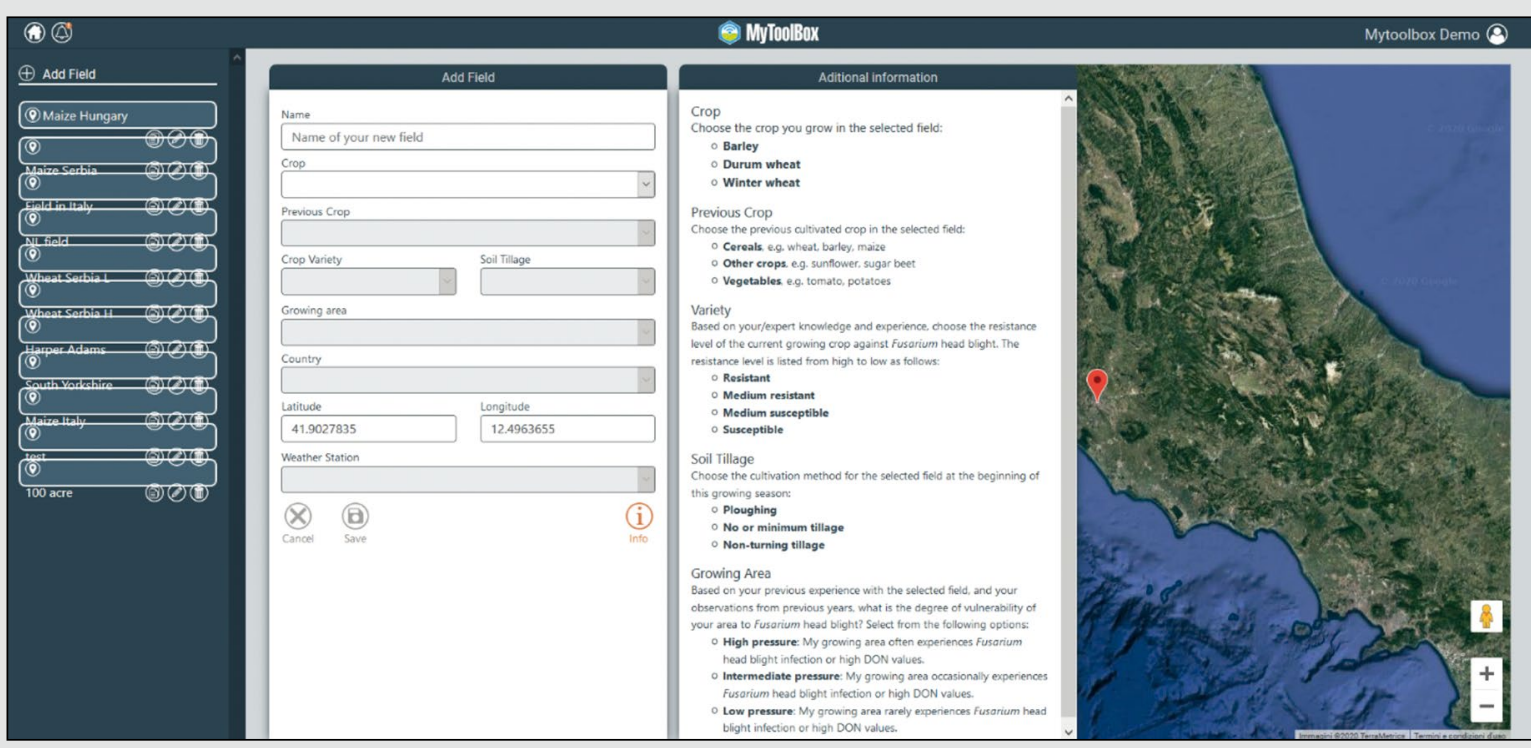

Figure 9. DSS pre-harvest forecasting tool: user input page.

red $(>1,250 \mu \mathrm{g} / \mathrm{kg})$. Based on the predicted mycotoxin risk class, the DSS provides the user with management advices.

For maize, once the user has chosen the location of his/ her field, a map of the whole country (in which the field is located) is shown. The grid is green or red on the basis of the model estimations for the mycotoxin contamination class. The user can select a map for either aflatoxins or fumonisins contamination of maize. For aflatoxins, the green class represents contamination $\leq 10 \mu \mathrm{g} / \mathrm{kg}$ and the red class is $>10 \mu \mathrm{g} / \mathrm{kg}$. For fumonisins the green class represents contamination $\leq 1000 \mu \mathrm{g} / \mathrm{kg}$ and the red class is $>1000 \mu \mathrm{g} / \mathrm{kg}$.

\section{Post-harvest silo management}

To use this dynamic post-harvest module, the user must purchase and install the sensor system in his/her grain silos, and connect the sensor network to the DSS (sensor data acquisition software). After logging in the dynamic silo management tool, the user can select the cereal being stored in the silo, see the list of his/her silos, and create a 


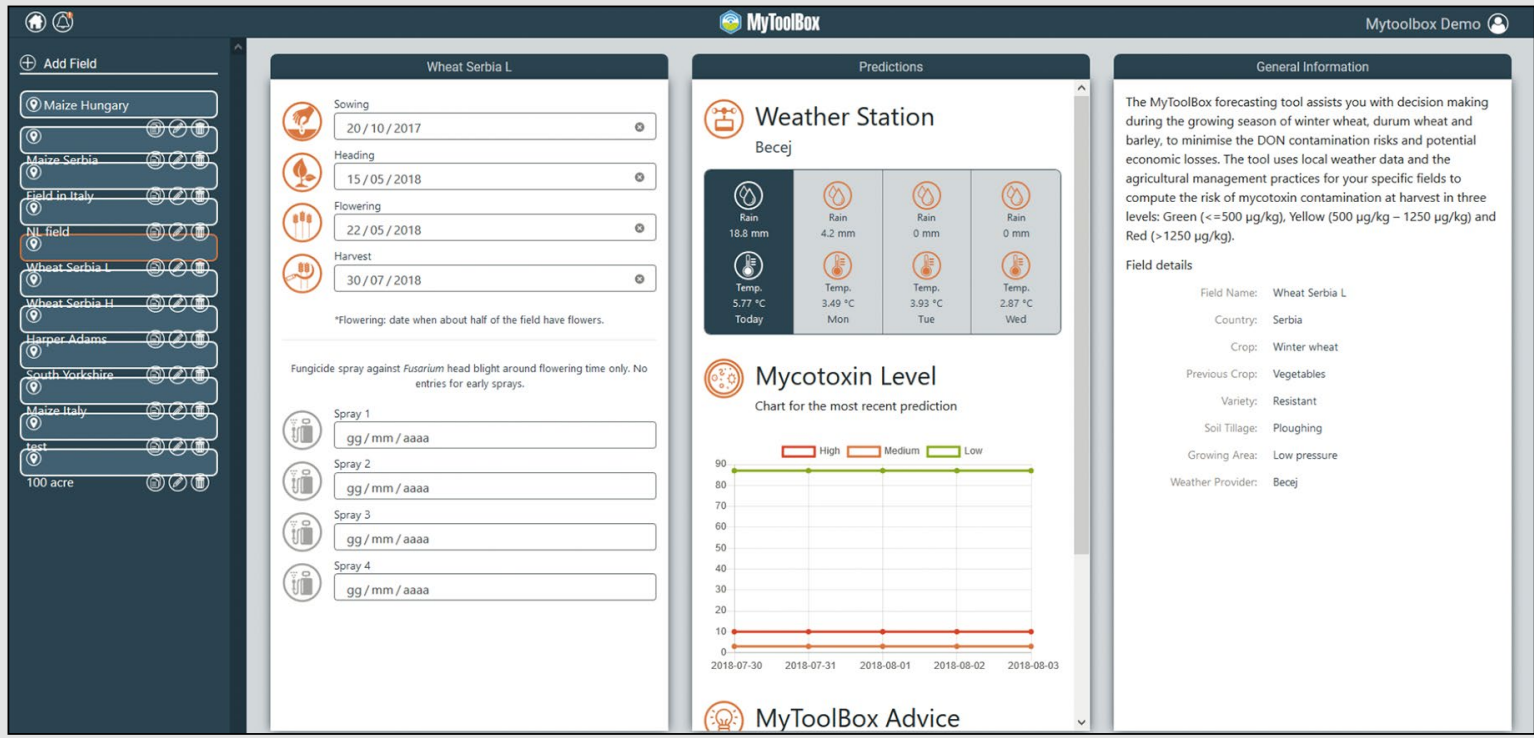

Figure 10. DSS pre-harvest forecasting tool: display forecasts for deoxynivalenol in wheat, durum wheat and barley.

new silo. When creating a new silo, the user must provide commodity type and the location of the new installation. The user can view his/her silos in the system: the strings of sensors are diagrammatically displayed in the DSS (Figure 11). By clicking on each sensor, the user can access the latest real-time data for temperature, $\mathrm{RH}$ and $\mathrm{CO}_{2}$. Predictions for fungal presence and mycotoxin contamination will be displayed for each sensor location. A colour code is used to identify, per sensor node, the risk for mould initiation and mycotoxins on the basis of the model output, and practical advice is given for intervention and remedial approaches for silo management, where necessary.
Results of the tests of the post-harvest dynamic module in silos in Italy and China revealed that the $\mathrm{CO}_{2}$ sensor provided an early warning of indicative changes, especially in a silo region where a simulated water incursion was introduced. The abiotic variables are linked to biological boundary models for mould growth and the predominant mycotoxin contamination in each commodity. This linkage enables real-time sensitivity and model accuracy for each of the chosen commodities.

These new hardware and software tools are a significant improvement for the tracking of the status of stored

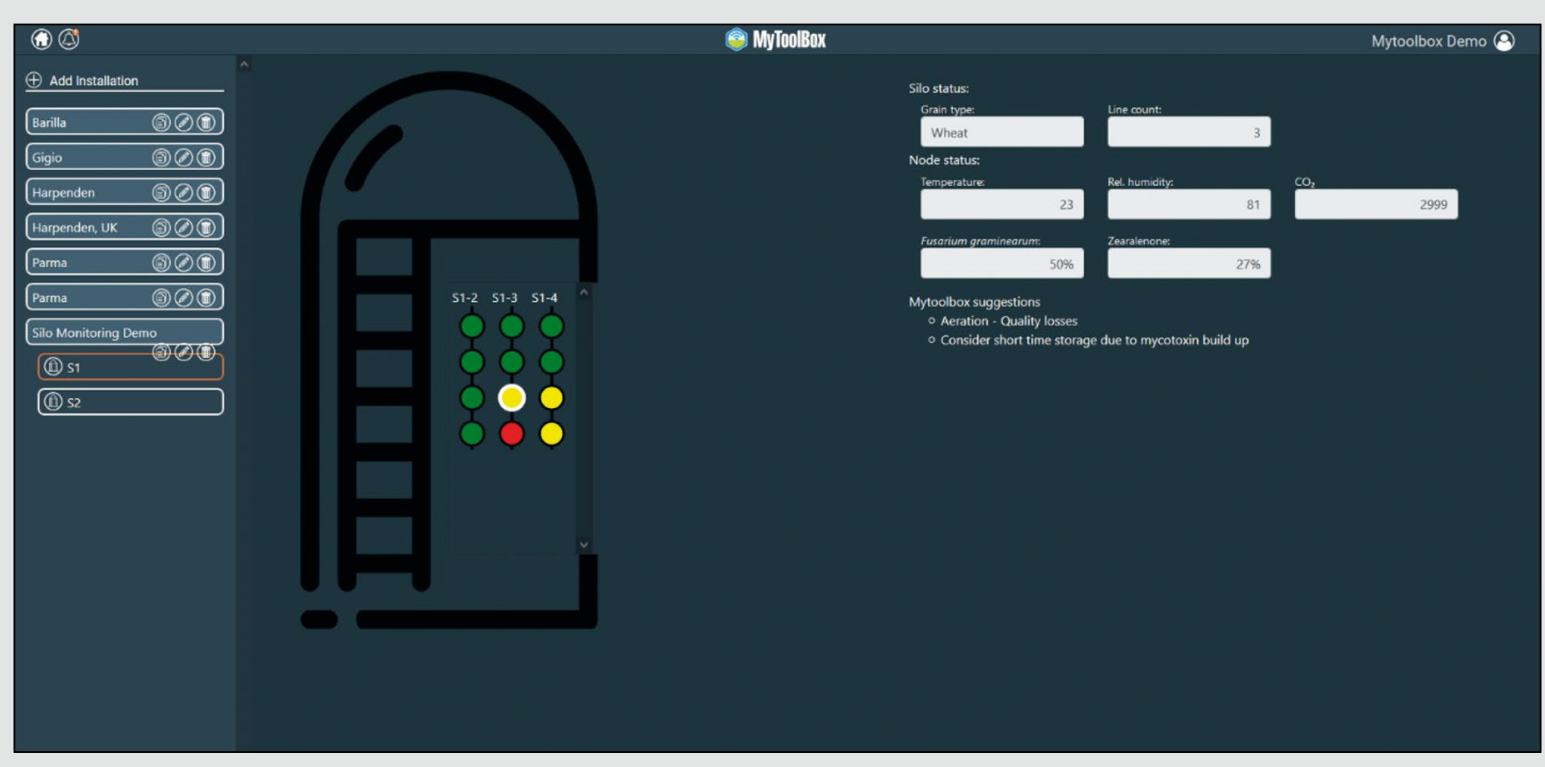

Figure 11. DSS silo monitoring tool: display results for coloured mycotoxin risk for each sensor on the three cables. 
commodities within silos. They provide early and real-time indications of problems and allow decisions to be made rapidly and quick remedial action to be taken to reduce economic losses due to mould growth and mycotoxins during storage.

\section{Conclusions}

This study developed an interactive and user-friendly DSS for mycotoxin prevention and control along the food and feed supply chain containing both static information modules and dynamic prediction modules. It is based on up-to-date information on mycotoxin management, which has been made available in an easy-to-understand series of guidelines and flowcharts as well as interactive tools and models. The developed DSS is unique, since no such complete and integrative DSS are available to date. Ultimately, the DSS will improve decision making on mycotoxin management by all actors along the supply chain and reduce mycotoxin contamination of the key economically important crops in Europe, thereby reducing economic losses and improving animal and human health.

\section{Acknowledgements}

This study was performed as part of the MyToolBox project which received funding from the European Union's Horizon 2020 research and innovation programme under grant agreement NO 678012. Additional financing from the Ministry of Agriculture, Nature and Food Quality in the Netherlands (via Topsector Agri-Food, project code AFEU-17018) is acknowledged.

\section{Conflict of interest}

The authors declare to have no competing interests.

\section{References}

Aldars-García, L., Ramos, A.J., Sanchis, V. and Marín, S., 2016. Modeling postharvest mycotoxins in foods: recent research. Current Opinion in Food Science 11: 46-50.

Alonso, V., Cavaglieri, L., Ramos, A., Torres, A. and Marin, S., 2017. Modelling the effect of $\mathrm{pH}$ and water activity in the growth of Aspergillus fumigatus isolated from corn silage. Journal of Applied Microbiology 122: 1048-1056.

American Peanut Council (APC), 2011. Good agricultural practices for safe growing and harvesting of peanuts. American Peanut Council Good Agricultural Practices, Washington, DC, USA.

Atanda, S., Aina, J., Agoda, S., Usanga, O. and Pessu, P., 2012. Mycotoxin management in agriculture: a review. Journal of Animal Science Advances 2: 250-260.
Battilani, P., Camardo Leggieri, M., Rossi, V. and Giorni, P., 2013. AFLA-maize, a mechanistic model for Aspergillus flavus infection and aflatoxin $\mathrm{B}_{1}$ contamination in maize. Computers and Electronics in Agriculture 94: 38-46.

Battilani, P. and Leggieri, M.C., 2015. Predictive modelling of aflatoxin contamination to support maize chain management. World Mycotoxin Journal 8: 161-170.

Birr, T., Verreet, J.-A. and Klink, H., 2019. Prediction of deoxynivalenol and zearalenone in winter wheat grain in a maize-free crop rotation based on cultivar susceptibility and meteorological factors. Journal of Plant Diseases and Protection 126: 13-27.

Bruns, H.A., 2003. Controlling aflatoxin and fumonisin in maize by crop management. Journal of Toxicology: Toxin Reviews 22: 153-173.

Chauhan, Y., Tatnell, J., Krosch, S., Karanja, J., Gnonlonfin, B., Wanjuki, I., Wainaina, J. and Harvey, J., 2015. An improved simulation model to predict pre-harvest aflatoxin risk in maize. Field Crops Research 178: 91-99.

Codex Alimentarius Commission (CAC), 2003. Code of practice for the prevention and reduction of mycotoxin contamination of cereals, including annexes on ochratoxin $\mathrm{A}$, zearalenone, fumonisins and trichothecenes. CAC/RCP: 51-2003. FAO/WHO, Rome, Italy.

Codex Alimentarius Commission (CAC), 2004. Code of practice for the prevention and reduction of aflatoxin contamination in peanuts. CAC/RCP 55. FAO/WHO, Rome, Italy.

Codex Alimentarius Commission (CAC), 2008. Code of practice for the prevention and reduction of aflatoxin contamination in dried figs. Doc. Ref. CAC/RCP: 65-2008. FAO/WHO, Rome, Italy.

Collins, M., Booth, B.B.B., Bhaskaran, B., Harris, G.R., Murphy, J.M., Sexton, D.M.H. and Webb, M.J., 2011. Climate model errors, feedbacks and forcings: a comparison of perturbed physics and multi-model ensembles. Climate Dynamics 36: 1737-1766.

De Rijk, T., Van Egmond, H., Van der Fels-Klerx, H.J., Herbes, R., De Nijs, M., Samson, R., Slate, A. and Van der Spiegel, M., 2015. A study of the 2013 Western European issue of aflatoxin contamination of maize from the Balkan area. World Mycotoxin Journal 8: 641-651.

Del Ponte, E., Fernandes, J. and Bergstrom, G., 2007. Influence of growth stage on Fusarium head blight and deoxynivalenol production in wheat. Journal of Phytopathology 155: 577-581.

Del Ponte, E.M., Fernandes, J.M.C. and Pavan, W., 2005. A risk infection simulation model for Fusarium head blight of wheat. Fitopatologia Brasileira 30: 634-642.

European Commission (EC), 2006a. Commission Recommendation 2006/576/EC of 17 August 2006 on the presence of deoxynivalenol, zearalenone, ochratoxin A, T-2 and HT-2 and fumonisins in products intended for animal feeding. Official Journal of the European Union L 229: 7-9.

European Commission (EC), 2006b. Commission Regulation (EC) No 401/2006 of 23 February 2006 laying down the methods of sampling and analysis for the official control of the levels of mycotoxins in foodstuffs. Official Journal of the European Union L 70: 12-34.

European Commission (EC), 2006c. Commission Regulation (EC) No 1881/2006. Official journal of the European Union L 364: 5-24. European Commission (EC), 2009. Commission Regulations (EC) No 152/2009 of 27 January 2009 laying down the methods of sampling and analysis for the official control of feed. Official journal of the European Union L 54: 3-132. 
European Commission (EC), 2016. Regulation (EU) 2016/679 of the European Parliament and of the Council of 27 April 2016 on the protection of natural persons with regard to the processing of personal data and on the free movement of such data, and repealing Directive 95/46/EC (General Data Protection Regulation). Official Journal of the European Union L 119: 1-88.

European Union Agency for Cybersecurity (ENISA), 2019. Pseudonymisation techniques and best practices - Recommendations on shaping technology according to data protection and privacy provisions. https://doi.org/10.2824/247711

Focker, M., Van der Fels-Klerx, H.J. and Oude Lansink, A., 2019. Costeffective sampling and analysis for mycotoxins in a cereal batch. Risk Analysis 39: 926-939.

Food Standards Agency, 2007. Code of good agricultural practice for the reduction of mycotoxins in UK cereals. Food Standards Agency, London, UK.

Franz, E., Booij, K. and van der Fels-Klerx, H.J., 2009. Prediction of deoxynivalenol content in Dutch winter wheat. Journal of Food Protection 72: 2170-2177.

Garcia-Cela, E., Kiaitsi, E., Sulyok, M., Krska, R., Medina, A., Petit Damico, I. and Magan, N., 2019. Influence of storage environment on maize grain: $\mathrm{CO}_{2}$ production, dry matter losses and aflatoxins contamination. Food Additives and Contaminants Part A 36: 175185.

Garcia-Cela, E., Kiaitsi, E., Sulyok, M., Medina, A. and Magan, N., 2018. Fusarium graminearum in stored wheat: use of $\mathrm{CO}_{2}$ production to quantify dry matter losses and relate this to relative risks of zearalenone contamination under interacting environmental conditions. Toxins 10: 86.

Garcia-Cela, E., Sanchez, F.G., Sulyok, M., Verheecke-Vaessen, C., Medina, A., Krska, R. and Magan, N., 2020. Carbon dioxide production as an indicator of Aspergillus flavus colonisation and aflatoxins/cyclopiazonic acid contamination in shelled peanuts stored under different interacting abiotic factors. Fungal Biology 124: 1-7.

Klem, K., Vanova, M., Hajslova, J., Lancová, K. and Sehnalová, M., 2007. A neural network model for prediction of deoxynivalenol content in wheat grain based on weather data and preceding crop. Plant Soil and Environment 53: 421.

Krska, R., De Nijs, M., McNerney, O., Pichler, M., Gilbert, J., Edwards, S., Suman, M., Magan, N., Rossi, V. and Van der FelsKlerx, H.J., 2016. Safe food and feed through an integrated toolbox for mycotoxin management: the MyToolBox approach. World Mycotoxin Journal 9: 487-495.

Landschoot, S., Waegeman, W., Audenaert, K., Haesaert, G. and De Baets, B., 2013a. Ordinal regression models for predicting deoxynivalenol in winter wheat. Plant Pathology 62: 1319-1329.

Landschoot, S., Waegeman, W., Audenaert, K., Van Damme, P., Vandepitte, J., De Baets, B. and Haesaert, G., 2013b. A fieldspecific web tool for the prediction of Fusarium head blight and deoxynivalenol content in Belgium. Computers and Electronics in Agriculture 93: 140-148.

Landschoot, S., Waegeman, W., Audenaert, K., Vandepitte, J., Haesaert, G. and De Baets, B., 2012. Toward a reliable evaluation of forecasting systems for plant diseases: a case study using Fusarium head blight of wheat. Plant Disease 96: 889-896.
Liu, C., Manstretta, V., Rossi, V. and Van der Fels-Klerx, H.J., 2018. Comparison of three modelling approaches for predicting deoxynivalenol contamination in winter wheat. Toxins 10: 267.

Liu, N., Liu, C., Dudaš, T., Loc, M., Bagi, F., and Van der Fels-Klerx, H.J., 2021. Improved aflatoxin and fumonisin forecasting models for maize (PREMA and PREFUM), using combined mechanistic and Bayesian network modelling - Serbia as a case study. Frontiers in Microbiology - Food Microbiology 12: 643604.

López-García, R., 2010. Mycotoxin management: an international challenge. Ensuring global food safety. Elsevier, New York, NY, USA, pp. 209-216.

Lopez-Garcia, R., Park, D. and Phillips, T., 1999. Integrated mycotoxin management systems. Food Nutrition and Agriculture 23: 38-48.

Maiorano, A., Reyneri, A., Sacco, D., Magni, A. and Ramponi, C., 2009. A dynamic risk assessment model (FUMAgrain) of fumonisin synthesis by Fusarium verticillioides in maize grain in Italy. Crop Protection 28: 243-256.

Mylona, K., Sulyok, M. and Magan, N., 2012. Fusarium graminearum and Fusarium verticillioides colonisation of wheat and maize, environmental factors, dry matter losses and mycotoxin production relevant to the EU legislative limits. Food Additives and Contaminants Part A 29: 1118-1128.

Rossi, V., Giosuè, S. and Delogu, G., 2003a. A model estimating risk for Fusarium mycotoxins in wheat kernels. Aspects of Applied Biology 68: 229-234.

Rossi, V., Giosuè, S., Pattori, E., Spanna, F. and Del Vecchio, A., 2003b. A model estimating the risk of Fusarium head blight on wheat. EPPO Bulletin 33: 421-425.

Rossi, V., Giosuè, S., Terzi, V. and Scudellari, D., 2007. A decision support system for Fusarium head blight on small grain cereals. EPPO Bulletin 37: 359-367.

Savić, Z., Dudaš, T., Loc, M., Grahovac, M., Budakov, D., Jajić, I., Krstović, S., Barošević, T., Krska, R. and Sulyok, M., 2020. Biological control of aflatoxin in maize grown in Serbia. Toxins 12: 162.

Schaafsma, A. and Hooker, D., 2007. Climatic models to predict occurrence of Fusarium toxins in wheat and maize. International Journal of Food Microbiology 119: 116-125.

Schwaber, K., 2004. Agile project management with Scrum. Pearson Education, Upper Saddle River, NJ, USA.

Schwaber, K. and Beedle, M., 2002. Agile software development with Scrum, 1. Prentice Hall, Upper Saddle River, NJ, USA.

Stadler, D., Sulyok, M., Schuhmacher, R., Berthiller, F. and Krska, R., 2018. The contribution of lot-to-lot variation to the measurement uncertainty of an LC-MS-based multi-mycotoxin assay. Analytical and Bioanalytical Chemistry 410: 4409-4418.

Tebaldi, C. and Knutti, R., 2007. The use of the multi-model ensemble in probabilistic climate projections. Philosophical Transactions of the Royal Society A 365: 2053-2075.

Van Asselt, E.D., Azambuja, W., Moretti, A., Kastelein, P., De Rijk, T.C., Stratakou, I. and Van der Fels-Klerx, H.J., 2012a. A Dutch field survey on fungal infection and mycotoxin concentrations in maize. Food Additives and Contaminants Part A 29: 1556-1565.

Van Asselt, E.D., Booij, C.J.H. and Van der Fels-Klerx, H.J., 2012 b. Modelling mycotoxin formation by Fusarium graminearum in maize in The Netherlands. Food Additives and Contaminants Part A 29: 1572-1580. 
Van Der Fels-Klerx, H.J., Burgers, S.L. and Booij, C., 2010a. Descriptive modelling to predict deoxynivalenol in winter wheat in the Netherlands. Food Additives and Contaminants Part A 27: 636-643.

Van der Fels-Klerx, H.J., Burgers, S.L.G.E. and Booij, C.J.H., 2010 b. Descriptive modelling to predict deoxynivalenol in winter wheat in the Netherlands. Food Additives and Contaminants Part A 27: 636-643.

Van der Fels-Klerx, H., De Rijk, T., Booij, C., Goedhart, P., Boers, E., Zhao, C., Waalwijk, C., Mol, H. and Van der Lee, T., 2012a. Occurrence of Fusarium head blight species and Fusarium mycotoxins in winter wheat in the Netherlands in 2009. Food Additives and Contaminants Part A 29: 1716-1726.
Van der Fels-Klerx, H.J., Olesen, J.E., Madsen, M. and Goedhart, P., 2012b. Climate change increases deoxynivalenol contamination of wheat in north-western Europe. Food Additives and Contaminants Part A 29: 1593-1604.

Van der Fels-Klerx, H.J., Goedhart, P.W., Elen, O., Borjesson, T., Hietaniemi, V. and Booij, C.J.H., 2012c. Modeling deoxynivalenol contamination of wheat in Northwestern Europe for climate change assessments. Journal of Food Protection 75: 1099-1106. 
